\title{
UHRF2 decreases H3K9ac expression by interacting with it through the PHD and SRA/YDG domain in HepG2 hepatocellular carcinoma cells
}

\author{
TING ZHANG ${ }^{1,2}$, LINGLIN ZHAO ${ }^{1,2}$, SHENGYUAN ZENG ${ }^{1,2}$, LU BAI $^{1,2}$, JUNXIA CHEN $^{1,2}$, \\ ZHENG ZHANG $^{1,2}$, YALAN WANG ${ }^{1,3}$ and CHANGZHU DUAN ${ }^{1,2}$ \\ ${ }^{1}$ Molecular Medicine and Cancer Research Center, Chongqing Medical University; Departments of ${ }^{2}$ Cell Biology and \\ Medical Genetics, and ${ }^{3}$ Pathology, Chongqing Medical University, Chongqing 400016, P.R. China
}

Received December 15, 2015; Accepted November 1, 2016

DOI: $10.3892 /$ ijmm.2016.2805

\begin{abstract}
Ubiquitin-like with PHD and ring finger domains 2 (UHRF2) is a multi-domain E3 ubiquitin ligase which is involved in epigenetic regulation and plays an essential role in tumorigenesis. However, the role of UHRF2 in histone H3 acetylation has not yet been fully elucidated and few studies have reported its role in hepatocellular carcinoma (HCC). In this study, we examined the correlation between UHRF2 and acetylated H3 in HCC. Immunohistochemistry and western blot analysis demonstrated that the levels of histone $\mathrm{H} 3$ lysine 9 acetylation (H3K9ac) and histone $\mathrm{H} 3$ lysine 14 acetylation (H3K14ac) were higher in the HCC tissues and HepG2 HCC cells compared with the adjacent non-tumor tissues and L02 normal cells. The level of UHRF2 was higher in the HCC tissues compared with the adjacent non-tumor tissues, but its expression did not exhibit a significant difference between the HepG2 HCC cells and the L02 normal cells. In addition, when comparing the HCC tissues, a higher expression of UHRF2 correlated with a lower expression of $\mathrm{H} 3 \mathrm{~K} 9 \mathrm{ac}$ in the $\mathrm{HCC}$ tissues. The overexpression of UHRF2 increased the expression of H3K9ac in L02 normal cells $(\mathrm{P}<0.01)$, but decreased the expression of $\mathrm{H} 3 \mathrm{~K} 9 \mathrm{ac}$ in HepG2 cancer cells $(\mathrm{P}<0.05)$. Moreover, immunofluorescence staining and co-immunoprecipitation assay indicated that UHRF2 co-localized and interacted with $\mathrm{H} 3 \mathrm{~K} 9 \mathrm{ac}$ in $\mathrm{L} 02$ and HepG2 cells and the plant homeodomain (PHD) finger domain was the key domain for UHRF2 directly binding to H3K9ac. Taken together, these results suggest that UHRF2 decreases the expression of $\mathrm{H} 3 \mathrm{~K} 9 \mathrm{ac}$ in HepG2 HCC cells and interacts with it through the PHD domain.
\end{abstract}

Correspondence to: Professor Changzhu Duan, Molecular Medicine and Cancer Research Center, Chongqing Medical University, 1 Yixueyuan Road, Chongqing 400016, P.R. China

E-mail: duanchzhu@cqmu.edu.cn

Key words: ubiquitin-like with PHD and ring finger domains 2, histone H3 lysine 9 acetylation, histone H3 lysine 14 acetylation, hepatocellular carcinoma, epigenetic regulation

\section{Introduction}

Hepatocellular carcinoma (HCC), the predominant form of adult liver malignancies, is one of the leading causes of cancer-related mortality worldwide $(1,2)$. According to a report by the World Health Organization (WHO) ('World Cancer Report 2014'), China now ranks first in the number of new cancer cases worldwide, and in particular, it ranks first in the number of new cases of HCC and related deaths worldwide. Currently, the incidence of liver cancer is approximately 25.7/100,000, becoming the type of cancer with the third largest mortality rate after gastric cancer and lung cancer. The detailed molecular mechanisms of hepatocarcinogenesis are not yet fully understood (3).

Histone acetylation occurs at the N-termini of the protein octamers and neutralizes the basic charge of the affected lysine (4). It is a reversible process, mediated by either histone acetyltransferases (HATs) or histone deacetylases (HDACs). It also plays a leading role in several cell functions and regulates numerous processes, including nucleosome assembly, chromatin condensation, folding, heterochromatin silencing and gene transcription (5). In recent years, histone $\mathrm{H} 3$ acetylation has become one of the hotspots in epigenetic regulation. It has been reported to be closely associated with the occurrence and development of multiple types of cancer and to also be related to the prognosis of multiple types of cancer, including HCC (6-9). The major acetylation sites of histone H3 tails are histone H3 lysine 9 (H3K9) and histone H3 lysine 14 (H3K14) and both of their modifications are associated with the promoters and enhancers of actively transcribed genes (10-12). The mechanisms responsible for the interaction of $\mathrm{H} 3 \mathrm{~K} 9 \mathrm{ac}$ and H3K14ac with ubiquitin-like with PHD and ring finger domains 2 (UHRF2) remain largely unknown.

UHRF2 is a multi-domain E3 ubiquitin ligase, which consists of an ubiquitin-like (UBL) domain, a tandem Tudor domain (TTD), a plant homeodomain (PHD) finger domain, a SET and RING associated YDG motif (SRA/YDG) domain, and a really interesting new gene (RING) finger domain (13). It has been reported that UHRF2 plays a very important role in cell cycle regulation through its association with multiple cell cycle-related proteins, including cyclins (A2, B1, D1 and E1), cyclin-dependent kinases (CDK2, CDK4 and CDK6), reti- 
noblastoma protein (pRB), p53 and proliferating cell nuclear antigen (PCNA) (13). It has also been reported that UHRF2 is involved in epigenetic regulation, including DNA methylation and histone modifications by interacting with hemi-methylated DNA, DNA methyltransferases (DNMT1, DNMT3a and DNMT3b), histone methyltransferase G9a, H3K9 methylation patterns (H3K9me2/me3) and HDAC1 (14-17). Therefore, we hypothesized that UHRF2 may be associated with H3K9ac and $\mathrm{H} 3 \mathrm{~K} 14 \mathrm{ac}$. In our previous studies, we demonstrated that UHRF2 interacted with hepatitis B virus (HBV) core protein and promoted its degradation (18), and we also demonstrated that UHRF2 inhibited the HBV replication cycle, not only through direct interaction with $\mathrm{HBc}$, but it also reduced the acetylation of HBV cccDNA-bound $\mathrm{H} 3$ histones (19). HBV is one of the major risk factors for $\mathrm{HCC}(1,3)$. These data suggest that UHRF2 may be associated with acetylated $\mathrm{H} 3$ and may play an important role in HCC.

In the present study, we demonstrated that the levels of $\mathrm{H} 3 \mathrm{~K} 9 \mathrm{ac}$ and H3K14ac were higher in HCC tissues and HepG2 cancer cells compared with adjacent non-tumor tissues and L02 normal cells. We also confirmed that a higher expression of UHRF2 correlated with a lower expression of H3K9ac in $\mathrm{HCC}$ tissues (when comparing HCC tissues) and that the overexpression of UHRF2 regulated the expression of $\mathrm{H} 3 \mathrm{~K} 9 \mathrm{ac}$ in different cells. Moreover, UHRF2 co-localized and interacted with H3K9ac in L02 and HepG2 cells, and UHRF2 interacted with H3K9ac through its PHD domain. Overall, these data reveal the molecular mechanisms responsible for the interaction of UHRF2 with acetylated $\mathrm{H} 3$ and provide new moleculer targets for HCC therapeutics.

\section{Materials and methods}

Reagents, cell lines and plasmids. Polyclonal rabbit human antiUHRF2/NIRF antibody (ab28673), anti-H3 (ab1791) antibody and monoclonal rabbit human antiH3K9ac (ab32129) antibody, H3K14ac (ab52946) were purchased from Abcam (Cambridge, MA, USA). Monoclonal mouse anti-Flag antibody (AF 519) and $\beta$-actin (AA128) were obtained from Beyotime Institute of Biotechnology (Jiangsu, China). Trichostatin A (TSA; T1952, $5 \mathrm{mM}$ in DMSO) was purchased from Sigma-Aldrich (St. Louis, MO, USA). Full-length and mutants UHRF2 plasmids were obtained by cloning the PCR fragment corresponding to UHRF2 into the pCMV-3xFlag vector (Fig. 1). The 293 normal renal epithelial cells, L02 normal liver cells, HepG2 HCC cells and pCMV-Flag-UHRF2, pCMV-Flag and deletion mutants of UHRF2 plasmids were stored by our laboratory.

Cell culture and transfection. The L02 cells were maintained in Roswell Park Memorial Institute-1640 (RPMI-1640; Gibco, Carlsbad, CA, USA), the 293 cells and HepG2 cells were maintained in Dulbecco's modified Eagle's medium (DMEM; HyClone Laboratories, Red Bank, NJ, USA). Both cells were supplemented with $10 \%$ fetal bovine serum (FBS; TBD Science Biotechnology, Tianjing, China) in a humidified atmosphere containing $5 \% \mathrm{CO}_{2}$ at $37^{\circ} \mathrm{C}$. Cell transfection (with pCMV-Flag-UHRF2 or pCMV-Flag) was carried out using Lipofectamine 2000 (Invitrogen, Carlsbad, CA, USA) according to the manufacturer's instructions. TSA $(400 \mathrm{nM})$ was added to the culture medium after 4-6 h of transfection and harvested at the end of $24 \mathrm{~h}$ if necessary. TSA is a HDACI and promotes the level of histone acetylation $(20,21)$. The cells were harvested at $24 \mathrm{~h}$ post-transfection.

Human tumor and non-neoplastic tissue. HCC tissues $(\mathrm{n}=5)$ and corresponding adjacent non-tumor liver tissues $(n=5)$ were obtained from patients at the Department of Pathology of the First Affiliated Hospital of Chongqing Medical University. Ethics approval for the study was obtained from the First Affiliated Hospital of Chongqing Medical University, and informed consent was obtained from each patient.

Immunohistochemistry. The tissues were deparaffinized for $1 \mathrm{~h}$ at $60^{\circ} \mathrm{C}$ in an oven and rehydrated by gradient alcohol. The antigen-retrieving steps were carried out in citrate buffer for $10 \mathrm{~min}$ in a microwave oven. The tissue sections were incubated overnight with primary antibodies to UHRF2 (1:200 dilution), H3 (1:200 dilution), H3K9ac (1:100 dilution) or H3K14ac (1:100 dilution) at $4^{\circ} \mathrm{C}$, and then incubated with the following secondary antibodies: biotinylated goat anti-rabbit IgG (SP9001) and biotinylated goat anti-mouse IgG (SP9002) (Zhongshan Goldenbridge Biotechnology, Corp., Beijing, China) for $15 \mathrm{~min}$ at $37^{\circ} \mathrm{C}$, using streptavidin/peroxidase (SP) histostain ${ }^{\mathrm{TM}}$-Plus kits, and stained with DAB (Zhongshan Goldenbridge Biotechnology Corp.). The nuclei were counterstained with hematoxylin. The rest of the procedure was performed according to manufacturer's instructions. Observations were performed using an Olympus multifunction microscope (Olympus, Tokyo, Japan). The staining intensity for UHRF2 was graded as 0 (no staining), 1 (mild staining), 2 (moderate staining) and 3 (intense staining). The staining extent was scored using the scale as follows: 0 (no staining of cells), 1 ( $<10 \%$ of tissue stained positive), 2 (10-50\% stained positive), 3 ( $>50 \%$ stained positive). The final staining score was defined as the sum of the intensity and extent scores. The specimens were divided into 3 groups according to their overall scores as follows: $0-2$, negative; $3-4$, weak staining (lower expression); 5-6, intense staining (higher expression).

Western blot analysis. For western blot analysis, the cells were washed 3 times with ice-cold phosphate-buffered saline (PBS) and lysed in RIPA buffer containing protease inhibitor cocktail (Roche Diagnostics Corp., Indianapolis, IN, USA) and phenylmethanesulfonyl fluoride (PMSF). Following incubation at $4^{\circ} \mathrm{C}$ for $30 \mathrm{~min}$, the lysate was centrifuged at $14,000 \mathrm{x} \mathrm{g}$ for $30 \mathrm{~min}$ at $4^{\circ} \mathrm{C}$. The protein concentration was determined using the bicinchoninic acid (BCA) protein assay kit (Beyotime Institute of Biotechnology) with BSA as the standard. Samples were denatured by $5 \mathrm{X} \mathrm{SDS}$ loading buffer for $5 \mathrm{~min}$ at $100^{\circ} \mathrm{C}$ and separated by sodium dodecyl sulfate-polyacrylamide gel electrophoresis (SDS-PAGE). Following electrotransfer, the membrane was probed with antibodies against UHRF2 $(1: 1,000$ dilution), $\beta$-actin (1:1,000 dilution), H3 (1:1,000 dilution), H3K9ac (1:1,000 dilution) or H3K14ac (1:500 dilution). The blots were then incubated with the secondary antibodies: HRP-labeled goat anti-mouse IgG (H+L) (A0192) and HRP-labeled goat anti-rabbit IgG (H+L) (A0208) (from Beyotime Institute of Biotechnology). Detection was carried out using the ECL chemiluminescent system (Merck Millipore KGaA, Darmstadt, Germany) according to the manufacturer's instructions. 

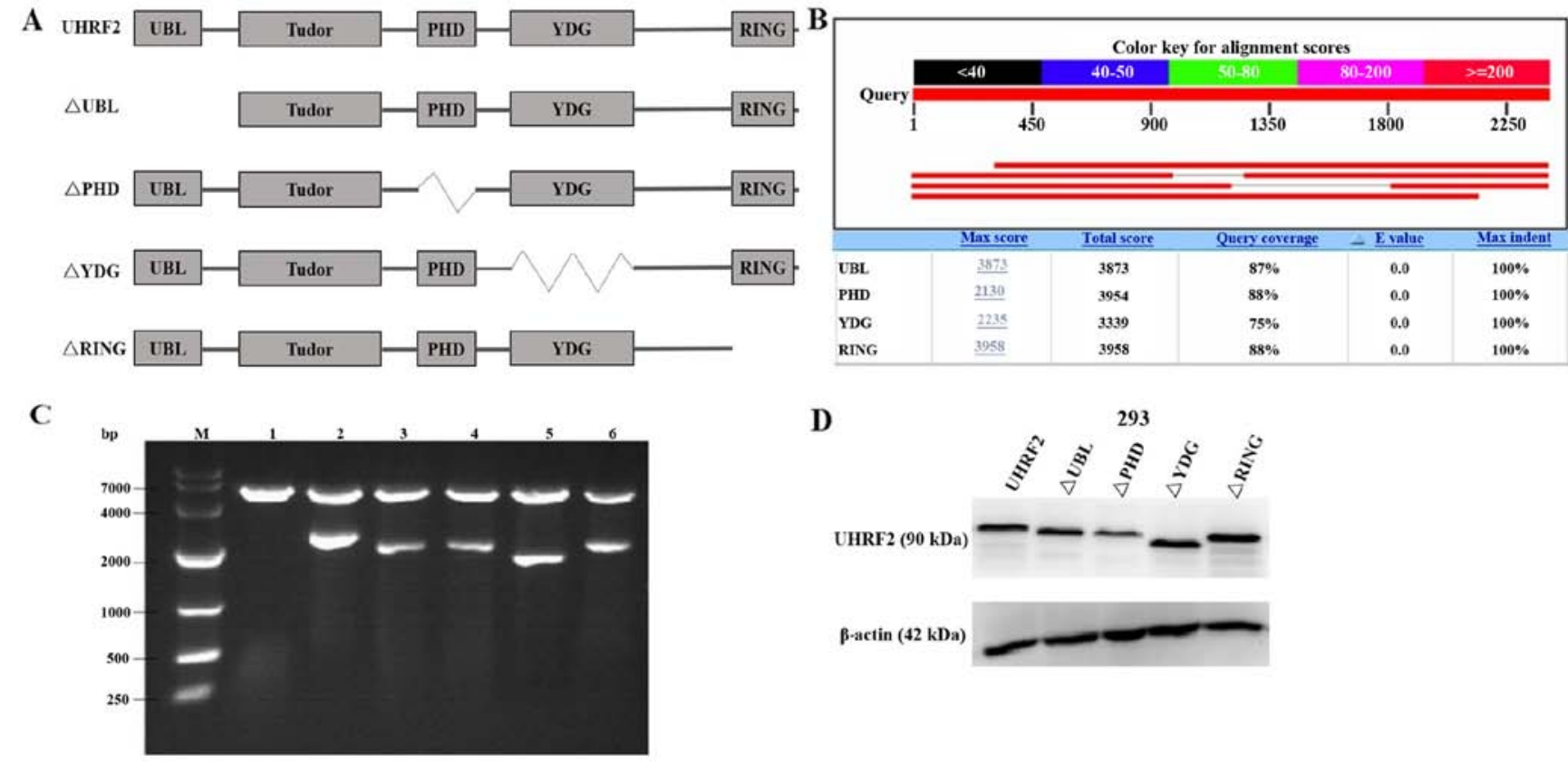

Figure 1. The deletion mutants of UHRF2. (A) Schematic presentation of domain deletion method of UHRF2. (B) BLAST results of sequenced inserts. (C) Identification of each recombinant plasmid by restriction enzyme digestion. Lane M is the DNA marker DL10000; lanes 1 to 6 are respective restriction maps of vector pCMV-3xFlag, Flag-UHRF2, Flag- $\Delta$ UBL, Flag- $\Delta$ PHD, Flag- $\Delta$ YDG and Flag- $\Delta$ RING. (D) 293 cells were transfected with the indicated plasmid. Western blot analysis demonstrated the deletion mutants of UHRF2 protein.

Co-immunoprecipitation (Co-IP) assay. For Co-IP, the L02 and HepG2 cells were collected at 24 h post-transfection, washed 3 times with ice-cold PBS and lysed with ice-cold NP-40-based lysis buffer containing protease inhibitor cocktail and PMSF for $30 \mathrm{~min}$, followed by centrifugation at $15,800 \mathrm{x} \mathrm{g}$ for $15 \mathrm{~min}$ at $4^{\circ} \mathrm{C}$. The protein concentration was determined using the $\mathrm{BCA}$ protein assay kit. The protein extracts were then incubated overnight with specific antibody against Flag and protein $\mathrm{G}$-agarose beads (Beyotime Institute of Biotechnology) were then added for $4 \mathrm{~h}$ at $4^{\circ} \mathrm{C}$. The immunoprecipitated complexes were washed 5 times using ice-cold lysis buffer, and bound proteins were released by boiling in $2 \mathrm{XSDS}$ loading buffer for $5 \mathrm{~min}$ at $100^{\circ} \mathrm{C}$. The precipitated proteins were then examined by western blot analysis. The input was used as a positive control.

Immunofluorescence staining. The L02 and HepG2 cells were grown on cover slips in 6-well plates for $24 \mathrm{~h}$ and transfected with pCMV-Flag-UHRF2. TSA was added to the culture medium at 4-6 h of transfection, and the cells were then incubated for a further $24 \mathrm{~h}$. The cells were washed 3 times with PBS, fixed with $4 \%$ paraformaldehyde for $30 \mathrm{~min}$, permeabilized in PBS containing $0.1 \%$ Triton X-100, and then washed and blocked with 5\% goat serum albumin in PBS for $30 \mathrm{~min}$ at room temperature to block non-specific antibodies and followed by incubation with antibodies against Flag (1:800 dilution), H3 (1:250 dilution), H3K9ac (1:250 dilution) or H3K14ac (1:250 dilution) overnight at $4^{\circ} \mathrm{C}$. After being washed 3 times with PBS, the cells were incubated with the secondary antibody, namely Alexa Fluor 488 goat anti-mouse IgG (ZF 0512; 1:100 dilution) or Alexa Fluor 594 goat anti-rabbit IgG (ZF 0516; 1:100 dilution) (Zhongshan Goldenbridge Biotechnology, Corp.) for $1 \mathrm{~h}$ at $37^{\circ} \mathrm{C}$ in the dark and then washed 3 times. The cell nuclei were stained with 4,6-diamidino-2-phenylindole (DAPI), washed
3 times again with PBS and finally sealed with $50 \%$ glycerin. Observations were performed under a laser scanning confocal microscope (Leica TCS-SP2, Leica, Wetzler, German).

Statistical analysis. The results are expressed as the means \pm standard deviation (SD). All data represent the means \pm SD of 3 separate experiments. Student's t-tests were used for statistical analysis. A value of $\mathrm{P}<0.05$ was considered to indicate a statistically significant difference.

\section{Results}

Endogenous expression of UHRF2, H3K9ac and H3K14ac in different cells and tissues. In our previous study, we demonstrated that UHRF2 decreased the acetylation of HBV cccDNA-bound H3 histones (19). In order to explore the molecular mechanisms responsible for the interaction between UHRF2 and acetylated H3, we first performed immunohistochemistry and western blot analysis to detect the endogenous expression levels of UHRF2, H3K9ac and H3K14ac in HCC tissues and adjacent non-tumor tissues, and in L02 and HepG2 cells, respectively. The results of immunohistochemistry revealed the negative staining of UHRF2 in the adjacent nontumor tissues, and the positive staining of UHRF2 in HCC tissues. In addition, the expression of H3K9ac and H3K14ac was higher in the HCC tissues compared to the adjacent non-tumor tissues (Fig. 2). The results of western blot analysis indicated that the protein level of UHRF2 did not exhibit a significant difference between the L02 normal cells and the HepG2 HCC cells; however, the protein levels of $\mathrm{H} 3 \mathrm{~K} 9 \mathrm{ac}$ were higher in the HepG2 cells than in the L02 cells $(\mathrm{P}<0.01)$ and the protein levels of H3K14ac were also higher in the HepG2 cells than in the L02 cells $(\mathrm{P}<0.05)$ (Fig. 3). These data indicated that the 

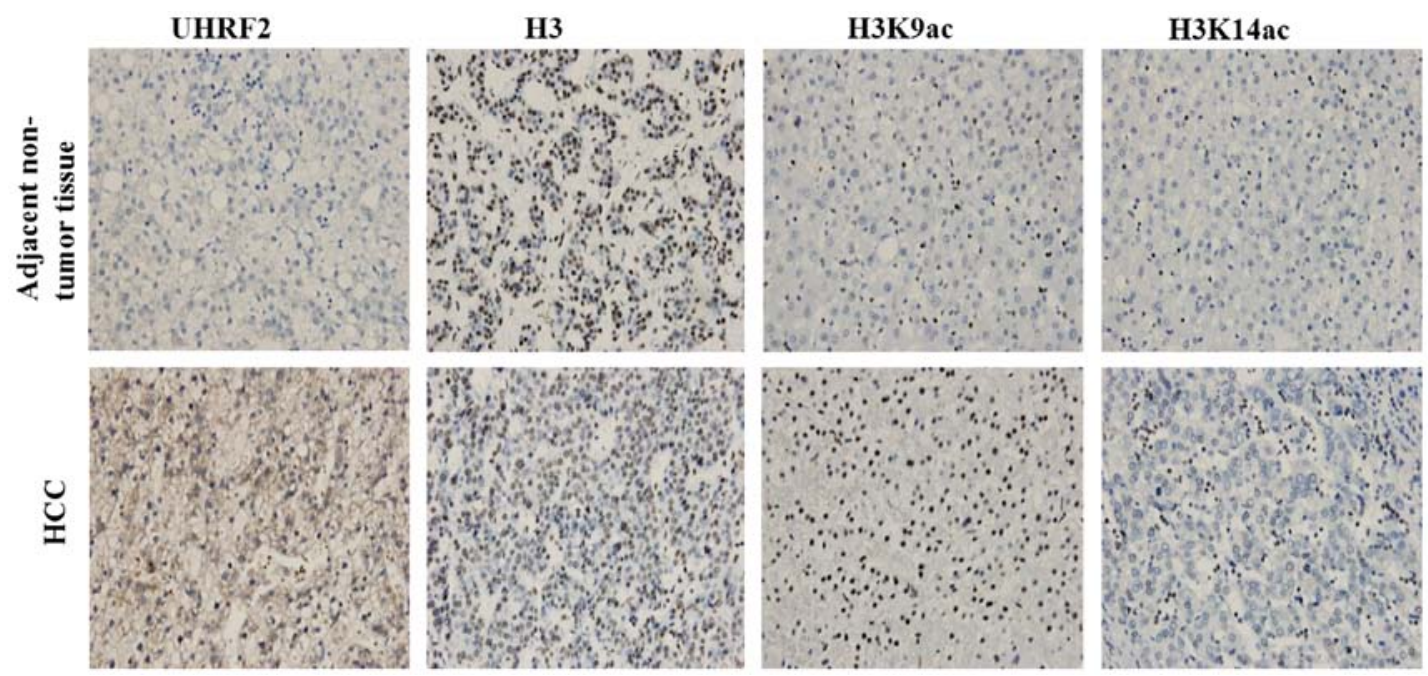

Figure 2. The expression levels of ubiquitin-like with PHD and ring finger domains 2 (UHRF2), histone H3 lysine 9 acetylation (H3K9ac) and histone H3 lysine 14 acetylation (H3K14ac) in different tissues. Immunohistochemistry of UHRF2, H3K9ac and H3K14ac expression in HCC tissues and adjacent nontumor tissues; the nuclei were counterstained with hematoxylin (magnification, $\mathrm{x} 400$ ).
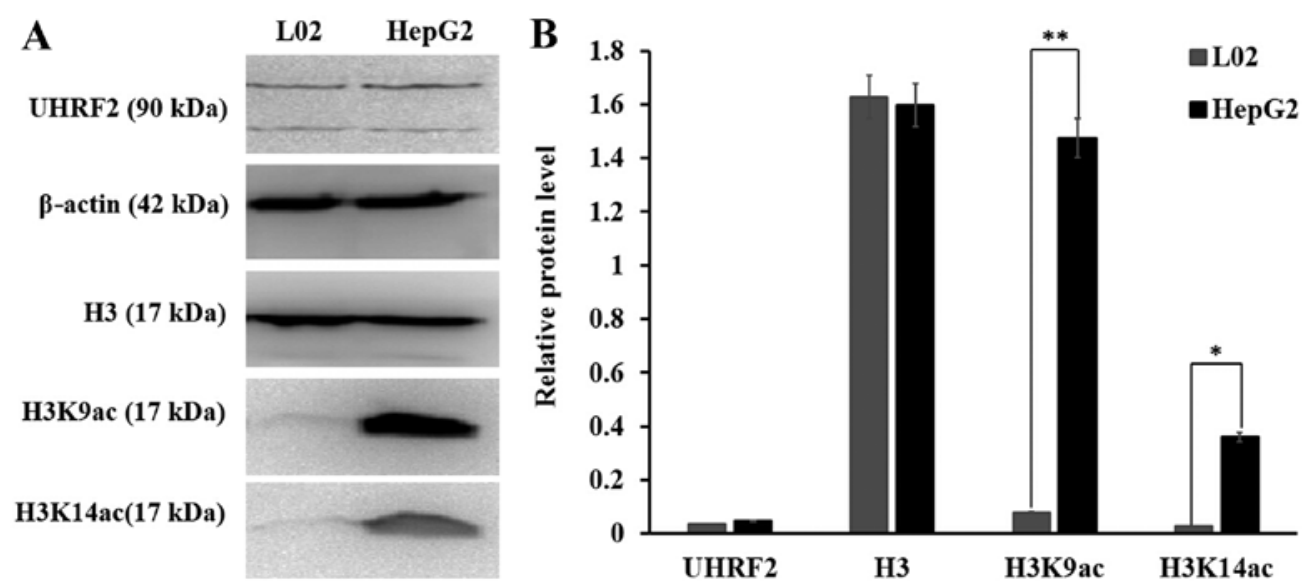

Figure 3. The protein levels of ubiquitin-like with PHD and ring finger domains 2 (UHRF2), histone H3 lysine 9 acetylation (H3K9ac) and histone H3 lysine 14 acetylation (H3K14ac) in different cells. (A) L02 normal cells and HepG2 hepatocellular carcinoma cells were harvested after $24 \mathrm{~h}$ and cell lysates were blotted with indicated antibodies. H3 and $\beta$-actin were used as a loading control. Western blot analysis demonstrated that H3K9ac and H3K14ac expression was higher in HepG2 cells compared with L02 cells. (B) Quantification of the expression data shown in (A). Data are expressed as the means $\pm \mathrm{SD}$, ( $\mathrm{n}=3$ experiments). ${ }^{* * *} \mathrm{P}<0.01$ and ${ }^{*} \mathrm{P}<0.05$, compared with the L02 cells.

protein level of UHRF2 was consistent with that of H3K9ac and H3K14ac in the HCC tissues; a higher expression of UHRF2 was associated with higher levels of $\mathrm{H} 3 \mathrm{~K} 9 \mathrm{ac}$ and $\mathrm{H} 3 \mathrm{~K} 14 \mathrm{ac}$ in HCC tissues when compared to adjacent non-tumor tissues.

A higher level of UHRF2 is associated with a lower level of H3K9ac in HCC tissues (comparison of different HCC tissues only). We then examined UHRF2, H3K9ac and H3K14ac expression between different HCC tissues only. We observed some interesting results regarding the expression of UHRF2 and H3K9ac in HCC tissues (Fig. 4). We found weak UHRF2 staining in some HCC tissues (lower expression of UHRF2; Fig. 4A), as well as intense UHRF2 staining in other HCC tissues (higher expression of UHRF2; Fig. 4B). The results of immunohistochemistry revealed that the expression of $\mathrm{H} 3 \mathrm{~K} 9 \mathrm{ac}$ was higher in the HCC tissues with a lower UHRF2 expression (Fig. 4A), but it was lower in the HCC tissues with a higher UHRF2 expression (Fig. 4B). The expression of $\mathrm{H} 3 \mathrm{~K} 9 \mathrm{ac}$ was higher in the $\mathrm{HCC}$ tissues with a lower UHRF2 expression compared with the HCC tissues with a higher UHRF2 expression. Thus, it appeared that a higher expression of UHRF2 correlated with a lower expression of H3K9ac when comparing the HCC tissues. H3K14ac staining did not exhibit any obvious difference between the HCC tissues (Fig. 4). These results demonstrated that the expression of UHRF2 negatively correlated with H3K9ac expression when comparing different HCC tissues.

Overexpression of UHRF2 effects on the expression of H3K9ac and H3K14ac. We then explored the molecular mechanisms responsible for the interaction between UHRF2 and acetylated $\mathrm{H} 3$ by upregulating UHRF2 expression by transfecting the cells with pCMV-Flag-UHRF2. Semi-quantitative analysis of the protein levels of $\mathrm{H} 3 \mathrm{~K} 9 \mathrm{ac}$ and $\mathrm{H} 3 \mathrm{~K} 14 \mathrm{ac}$ was performed by western blot analysis. The overexpression of UHRF2 increased the level of H3K9ac in the L02 cells compared to the cells transfected with the empty vector $(\mathrm{P}<0.01$; Fig. $5 \mathrm{~A}$ and $\mathrm{B})$; however, it decreased 
A

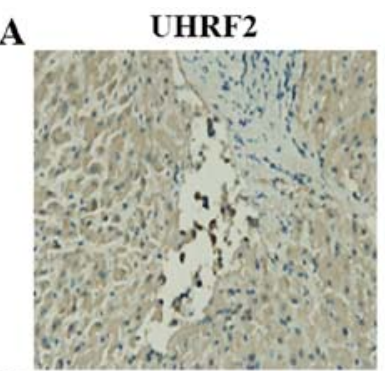

B

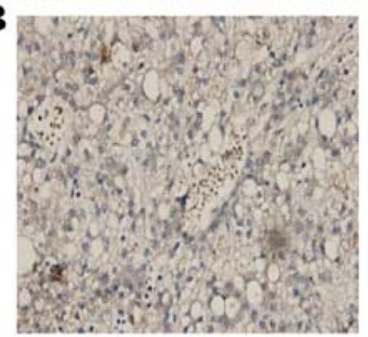

H3
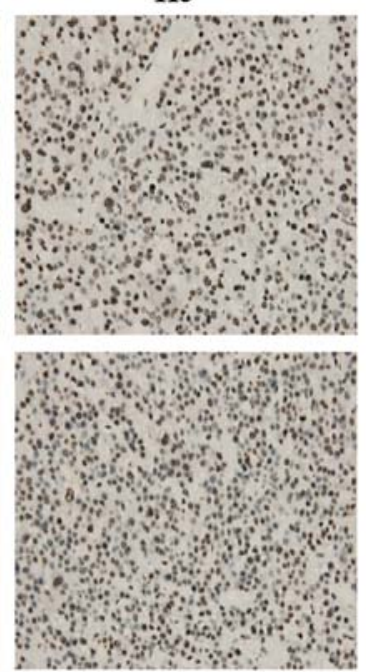

H3K9ac
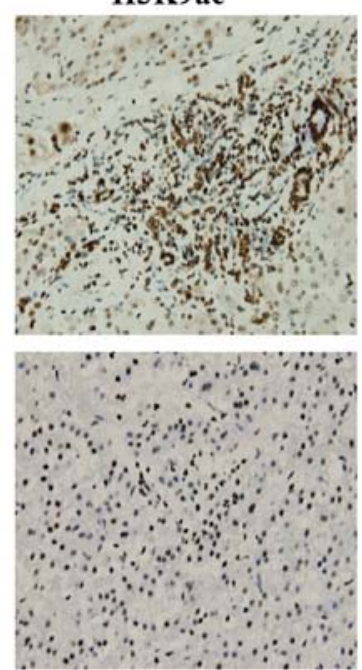

H3K14ac
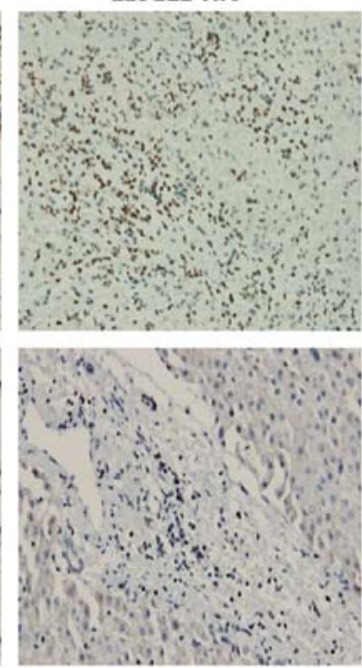

Figure 4. A higher level of ubiquitin-like with PHD and ring finger domains 2 (UHRF2) is associated with a lower level of histone H3 lysine 9 acetylation (H3K9ac) in hepatocellular carcinoma (HCC) tissues. (A and B) For immunohistochemistry, the HCC tissue sections were stained with the indicated antibodies; the nuclei were counterstained with hematoxylin (magnification, x400). (A) HCC tissues with a lower UHRF2 expression; (B) HCC tissues with a higher UHRF2 expression.

A

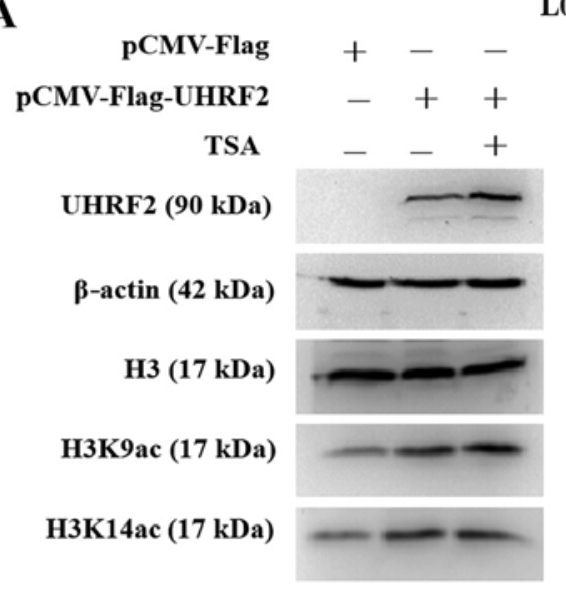

C

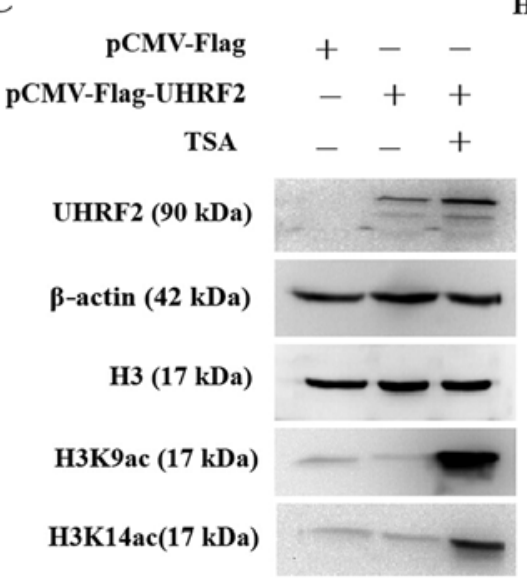

B

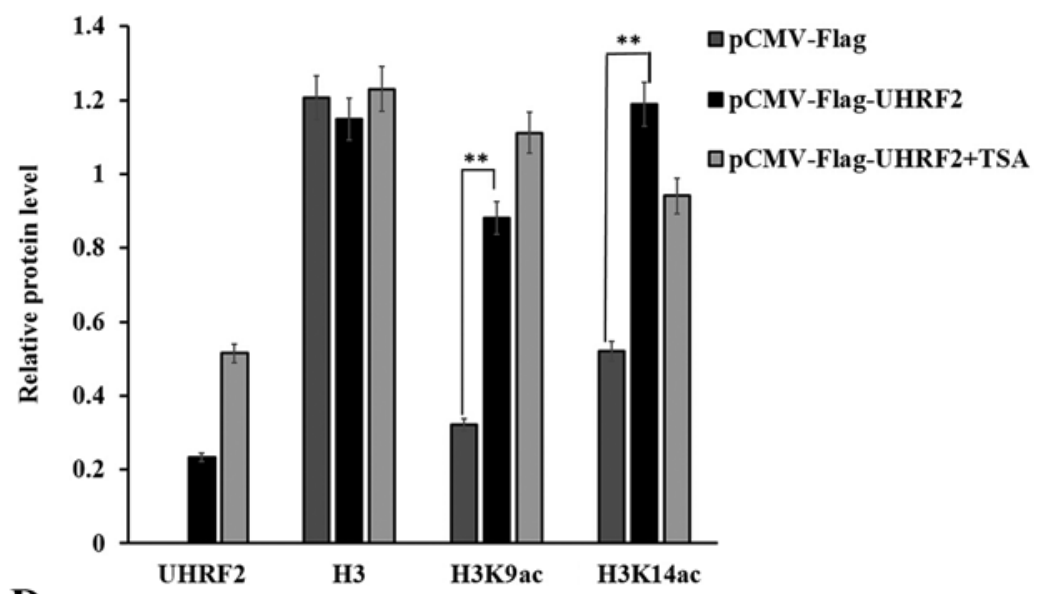

D

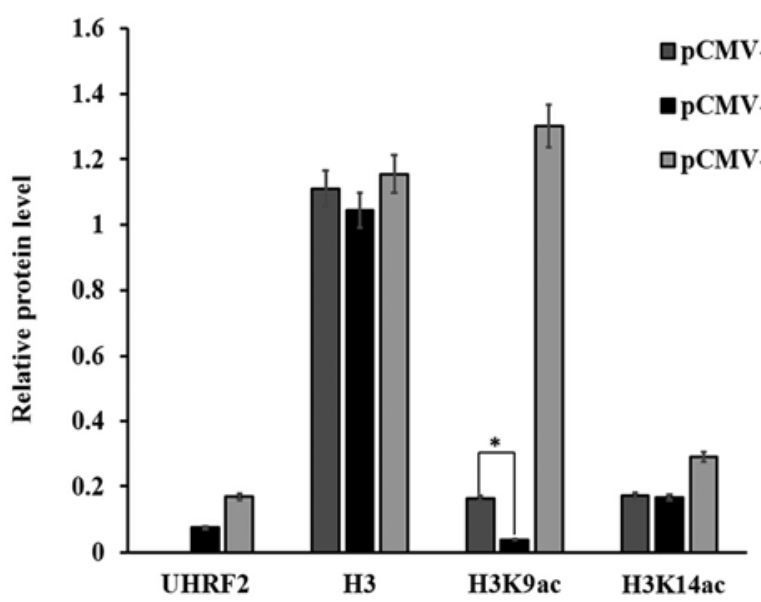

HepG2 

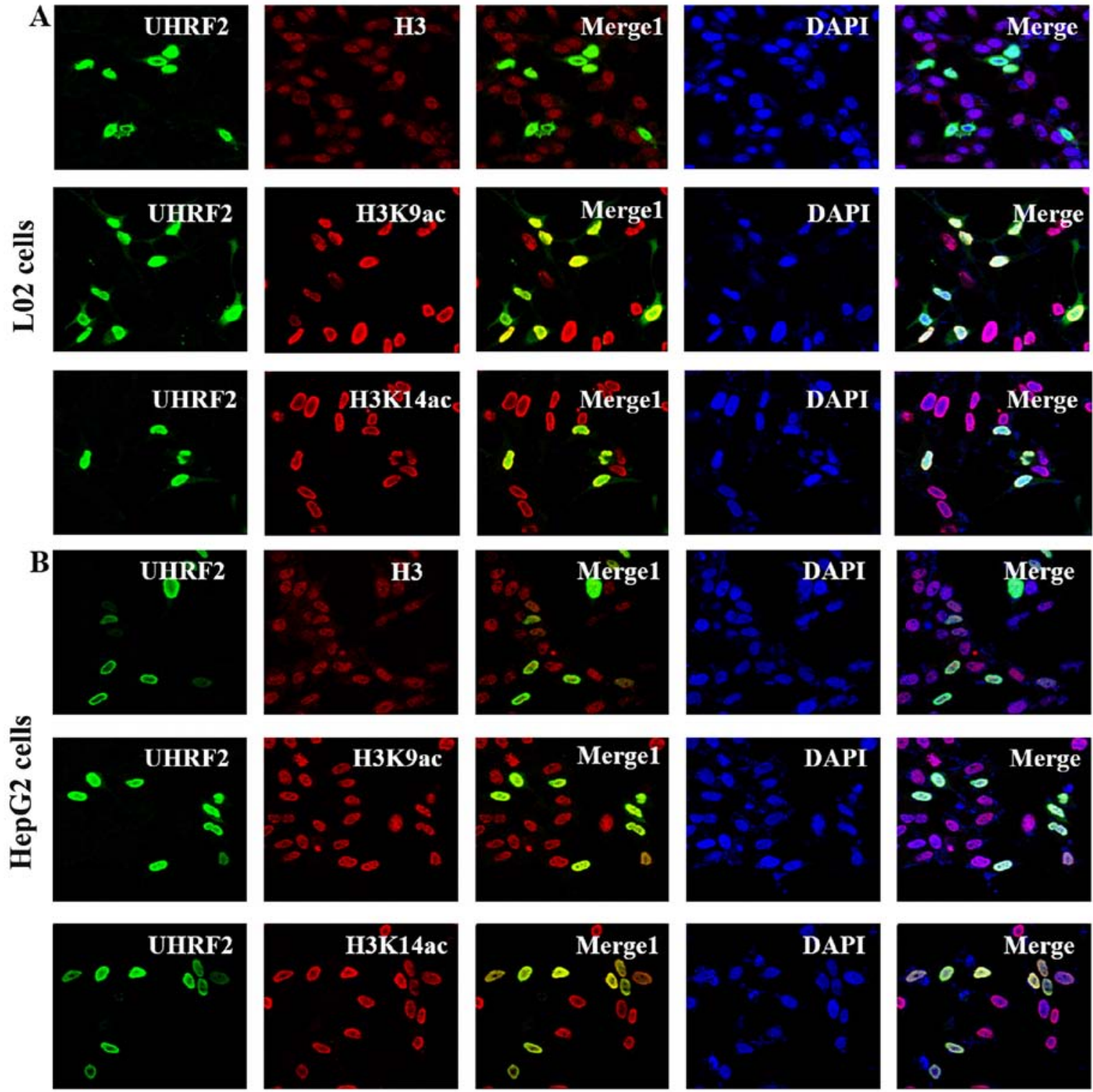

Figure 6. Co-localization of ubiquitin-like with PHD and ring finger domains 2 (UHRF2) and H3, histone H3 lysine 9 acetylation (H3K9ac) and histone H3 lysine 14 acetylation (H3K14ac) as shown under a laser scanning confocal microscope. (A and B) L02 normal cells and HepG2 hepatocellular carcinoma (HCC) cells were transfected with pCMV-Flag-UHRF2. Immunofluorescence staining of UHRF2 (green) and H3, H3K9ac and H3K14ac (red) is indicated in the cells. The DAPI (blue) staining represents the nucleus. The merge images (yellow) demonstrated that UHRF2 co-localized with H3, H3K9ac and H3K14ac in the nucleus in vitro (magnification, $\mathrm{x} 400$ ).

the level of $\mathrm{H} 3 \mathrm{~K} 9 \mathrm{ac}$ in the HepG2 cells (P<0.05; Fig. 5C and D). In addition, the overexpression of UHRF2 increased the level of $\mathrm{H} 3 \mathrm{~K} 14 \mathrm{ac}$ in the L02 cells, whereas it did not significantly alter its level in the HepG2 cells $(\mathrm{P}<0.01)$ (Fig. 5). These results indicated that the overexpression of UHRF2 increased the expression of $\mathrm{H} 3 \mathrm{~K} 14 \mathrm{ac}$ in L02 normal cells, but it did not significantly alter the expression of H3K14ac in HepG2 cells, whereas it had a differential effect on the expression of H3K9ac in L02 normal cells as compared with HepG2 HCC cells.

UHRF2 co-localizes with H3, H3K9ac and H3K14ac. To investigate the regulatory effect of UHRF2 on the expression of H3K9ac and H3K14ac, the subcellular localization of UHRF2, H3, H3K9ac and H3K14ac was detected in the L02 and HepG2 cells by immunofluorescence staining with a laser scanning confocal microscope. UHRF2 is an essential protein for the maintenance of methylated histone $\mathrm{H} 3$ lysine 9 (H3K9me) $(14,17)$. We hypothesized that UHRF2 may responsible for recruiting $\mathrm{H} 3 \mathrm{~K} 9 \mathrm{ac}$ and $\mathrm{H} 3 \mathrm{~K} 14 \mathrm{ac}$. The results of immunofluorescence staining indicated that UHRF2 was localized in the nucleus (green). H3, H3K9ac and H3K14ac exhibited nucelar distribution (red) (Fig. 6). More significantly, in the merged images in Fig. 6, a yellow area (Merge1) could be observed, which indicated that there was a co-localization of UHRF2 with H3, H3K9ac and H3K14ac in both the L02 and HepG2 cells (Fig. 6).

UHRF 2 interacts with H3K9ac but not H3K14ac. To further investigate the interaction between UHRF2 and acetylated $\mathrm{H} 3$, we performed Co-IP assay. The cells were transfected with 

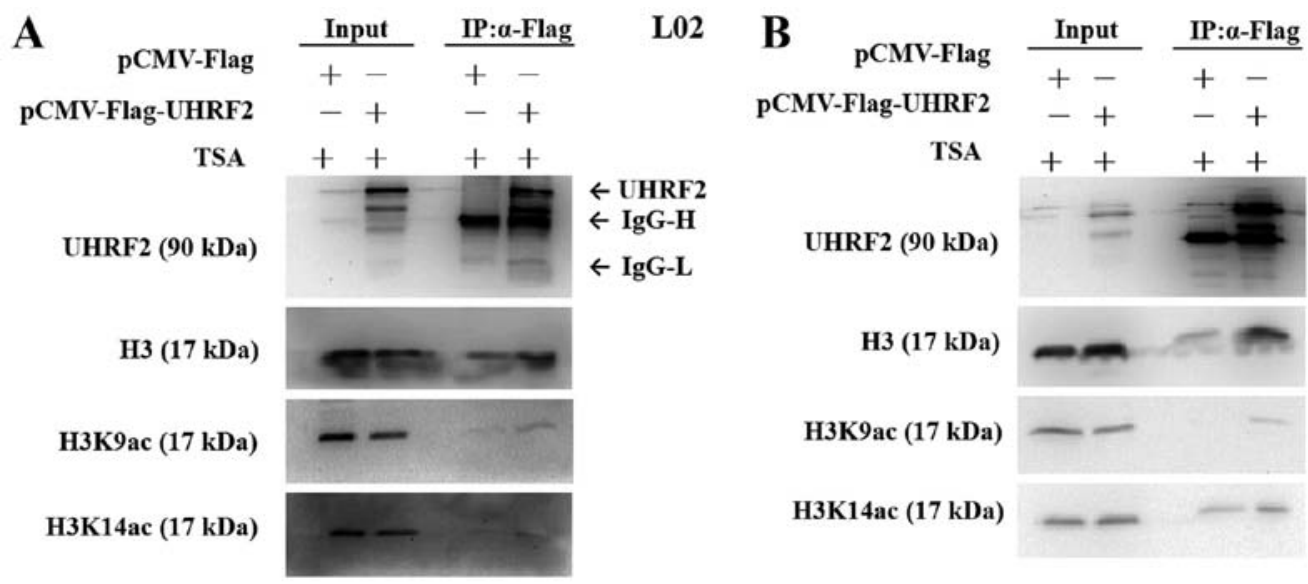

Figure 7. Interaction between ubiquitin-like with PHD and ring finger domains 2 (UHRF2) and histone H3 lysine 9 acetylation (H3K9ac). (A and B) Immunoprecipitation of H3K9ac and histone H3 lysine 14 acetylation (H3K14ac) with UHRF2. L02 and HepG2 cells were transfected with pCMVFlag-UHRF2 or pCMV-Flag (mock vector), TSA (400 nM) was added to the culture medium and cells were harvested at the end of $24 \mathrm{~h}$. Cell extracts were prepared and immunoprecipitated with anti-Flag antibody and protein G-agarose beads, then input (10\% whole cell lysates) and immunoprecipitates (IP) were probed by western blot analysis with indicated antibodies.

pCMV-Flag-UHRF2 or pCMV-Flag (mock vector). Flag antibody and protein G-agarose beads were used to co-immunoprecipitate $\mathrm{H} 3$, H3K9ac, H3K14ac protein from the L02 and HepG2 cell extracts. The results revealed that only the precipitation of Flag-UHRF2-interacting protein complexes from cells transfected with pCMV-Flag-UHRF2 could detect the expression of $\mathrm{H} 3 \mathrm{~K} 9 \mathrm{ac}$, indicating that $\mathrm{H} 3 \mathrm{~K} 9 \mathrm{ac}$ was indeed efficiently precipitated with UHRF2 (Fig. 7). However, H3K14ac was not detected in the L02 cells (Fig. 7A), but it was detected in both the pCMV-Flag-UHRF2- and pCMV-Flag-transfected HepG2 HCC cells, indicating that $\mathrm{H} 3 \mathrm{~K} 14 \mathrm{ac}$ could not be precipitated with UHRF2 (Fig. 7B).

The PHD domain of UHRF2 is important for H3K9ac binding. UHRF2 is a multi-domain protein (Fig. 1), its PHD domain has been showed to read part of the histone code (16) and it is characterized as a versatile epigenetic reader, linking posttranslational modifications (PTMs), particularly the histone $\mathrm{H} 3$ tail (22). In order to find the required domains of UHRF2 interacting with $\mathrm{H} 3 \mathrm{~K} 9 \mathrm{ac}$, we performed IP assay of the cells transfected with deletion mutants of UHRF2 plasmids or pCMV-Flag. The results revealed that H3K9ac expression was significantly lower compared with the input at the PHD deletion mutant domain, indicating that the PHD domain was the key domain for the interaction of UHRF2 with H3K9ac (Fig. 8). Of note, H3K9ac expression was significantly lower compared with the input at both the PHD deletion mutant domain and SRA/YDG deletion mutant domain in the HepG2 HCC cells, indicating not only the PHD domain, but also the SRA/YDG domain were important for the interaction of UHRF2 with H3K9ac in HepG2 HCC cells (Fig. 8B).

\section{Discussion}

UHRF2 had been proposed as a potential tumor suppressor in breast cancer cell lines and A549 lung cancer cells $(23,24)$, but has been shown to play an oncongenic role in colorectal cancer and glioma cells (25-27). The expression UHRF2 differs in different types of cancer, illustrating the heterogeneity of cancer, and has also been suggested that UHRF2 plays an important role in tumorigenesis. However, few studies have reported the role of UHRF2 in HCC. In this study, demonstrated that the expression of UHRF2 was higher in HCC tissues compared with adjacent non-tumor tissues. Accumulating evidence has established that tumor-associated epigenetic alterations, including DNA methylation and histone modifications are important determinants in the initiation and progression of $\mathrm{HCC}$ and represent promising biomarkers and therapeutic targets $(1,3,9,28)$. A high expression of trimethylated histone $\mathrm{H} 3$ lysine 4 has been shown to be associated with a poor prognosis in $\mathrm{HCC}$, and the increased acetylation of $\mathrm{H} 3 \mathrm{~K} 9$ has been associated with increased $\mathrm{H} 3 \mathrm{~K} 4$ methylation $(7,29)$. This study also demonstrated that the levels of H3K9ac and H3K14ac were higher in HepG2 HCC cells and HCC tissues compared with L02 normal cells and adjacent nontumor tissues. Moreover, the first evidence was provided that the overexpression of UHRF2 downregulated the protein level of $\mathrm{H} 3 \mathrm{~K} 9 \mathrm{ac}$ in HepG2 cancer cells, but upregulated the protein level of H3K9ac in L02 normal cells. A higher level of UHRF2 was associated with a lower level of $\mathrm{H} 3 \mathrm{~K} 9 \mathrm{ac}$ when comparing HCC tissues. Therefore, UHRF2 and H3K9ac together may serve as a biological marker for HCC therapeutics.

UHRF2 is involved in several important epigenetic modifications, including DNA methylation, histone methylation and acetylation $(14,17,26,30)$. In this study, we first reported that UHRF2 co-localized with H3K9ac and H3K14ac. In addition, UHRF2 interacted with $\mathrm{H} 3 \mathrm{~K} 9 \mathrm{ac}$, but not H3K14ac, and this result indicated site binding specificity when UHRF2 combined with acetylated H3. When a specific site of acetylated histones combines with UHRF2, this triggers a specific event downstream. UHRF1, another member of the UHRF family of proteins, is highly similar to UHRF2 in both sequence and structure, and interacts with methylated H3K9 through the PHD domain (16). UHRF2 specifically binds to H3K9me2/3 at the TTD-PHD region (17). The crystal structure basically displays that the PHD domain interacts with the H3 N-terminal tail $(22,31)$. In this study, we clarified that the PHD domain of UHRF2 is the key region for its interaction with $\mathrm{H} 3 \mathrm{~K} 9 \mathrm{ac}$. These results revealed the crosstalk 
A

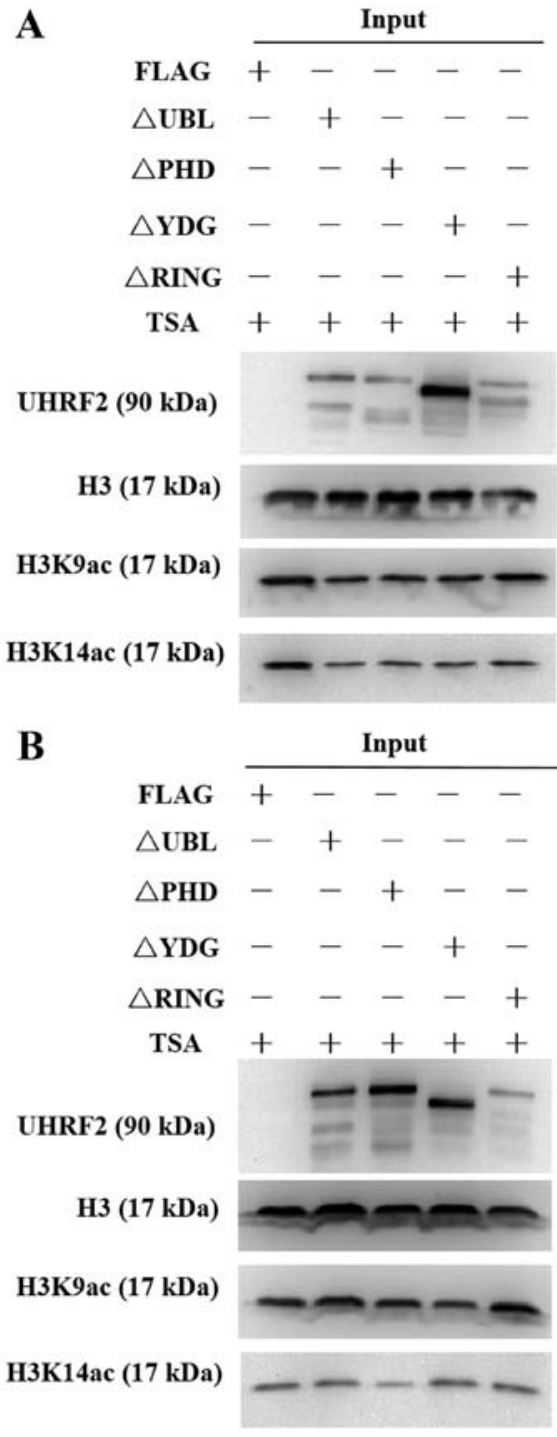

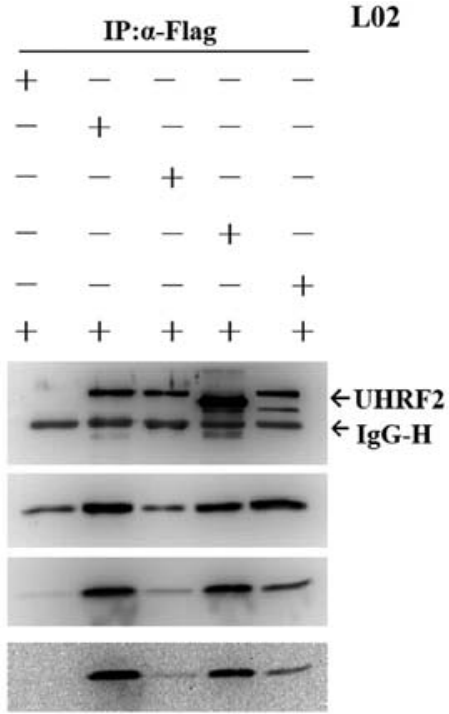

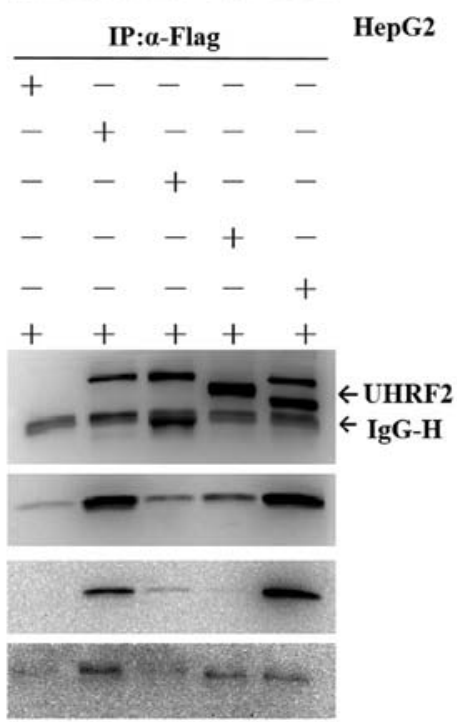

Figure 8. PHD domain is the key region for ubiquitin-like with PHD and ring finger domains 2 (UHRF2) binding to histone H3 lysine 9 acetylation (H3K9ac). (A and B) L02 and HepG2 were transfected with deletion mutants of UHRF2 or pCMV-Flag (mock vector), TSA (400 nM) was added to the culture medium and cells were harvested at the end of $24 \mathrm{~h}$. Cell extracts were prepared and immunoprecipitated with anti-Flag antibody and protein G-agarose beads followed by western blot analysis with indicated antibodies. These results showed that UHRF2 interacted with H3K9ac and H3K14ac through its PHD domain.

and the mechanistic link between UHRF2 and acetylated H3. Furthermore, it has been reported that the SRA/YDG domain is essential for binding histone $\mathrm{H} 3$ tails (32). We also found that the SRA/YDG domain was also required in combining with H3K9ac in HepG2 cells, unlike in L02 normal cells. Further studies are required in order to determine the difference in the required domain in normal and cancer cells, as regards UHRF2 binding to H3K9ac.

In conclusion, our findings demonstrated that the overexpression of UHRF2 decreased the protein level of H3K9ac in HepG2 cancer cells, but it increased the protein level of H3K9ac in L02 normal cells, and a higher level of UHRF2 expression was associated with a lower level of $\mathrm{H} 3 \mathrm{~K} 9 \mathrm{ac}$ expression in $\mathrm{HCC}$ tissues. Moreover, our findings also demonstrated that UHRF2 interacted with $\mathrm{H} 3 \mathrm{~K} 9 \mathrm{ac}$ directly and the PHD domain was the key domain for the binding of UHRF2 to H3K9ac. These results may provide a better understanding of the crosstalk between UHRF2 and H3K9ac, and highlight the possibilities that UHRF2 and H3K9ac together may be therapeutic target in HCC.

\section{References}

1. Ma L, Chua MS, Andrisani O and So S: Epigenetics in hepatocellular carcinoma: An update and future therapy perspectives. World J Gastroenterol 20: 333-345, 2014.

2. Siegel R, Naishadham D and Jemal A: Cancer statistics, 2012. CA Cancer J Clin 62: 10-29, 2012.

3. Puszyk WM, Trinh TL, Chapple SJ and Liu C: Linking metabolism and epigenetic regulation in development of hepatocellular carcinoma. Lab Invest 93: 983-990, 2013.

4. Kratz A, Arner E, Saito R, Kubosaki A, Kawai J, Suzuki H, Carninci P, Arakawa T, Tomita M, Hayashizaki Y and Daub CO: Core promoter structure and genomic context reflect histone 3 lysine 9 acetylation patterns. BMC Genomics 11: 257, 2010.

5. Shahbazian MD and Grunstein M: Functions of site-specific histone acetylation and deacetylation. Annu Rev Biochem 76: 75-100, 2007.

6. Elsheikh SE, Green AR, Rakha EA, Powe DG, Ahmed RA, Collins HM, Soria D, Garibaldi JM, Paish CE, Ammar AA, et al: Global histone modifications in breast cancer correlate with tumor phenotypes, prognostic factors, and patient outcome. Cancer Res 69: 3802-3809, 2009.

7. He C, Xu J, Zhang J, Xie D, Ye H, Xiao Z, Cai M, Xu K, Zeng Y, $\mathrm{Li} \mathrm{H}$ and Wang J: High expression of trimethylated histone $\mathrm{H} 3$ lysine 4 is associated with poor prognosis in hepatocellular carcinoma. Hum Pathol 43: 1425-1435, 2012. 
8. Neureiter D, Jäger T, Ocker M and Kiesslich T: Epigenetics and pancreatic cancer: Pathophysiology and novel treatment aspects. World J Gastroenterol 20: 7830-7848, 2014.

9. Hung SY, Lin HH, Yeh KT and Chang JG: Histone-modifying genes as biomarkers in hepatocellular carcinoma. Int J Clin Exp Pathol 7: 2496-2507, 2014.

10. Karmodiya K, Krebs AR, Oulad-Abdelghani M, Kimura $\mathrm{H}$ and Tora L: H3K9 and H3K14 acetylation co-occur at many gene regulatory elements, while $\mathrm{H} 3 \mathrm{~K} 14 \mathrm{ac}$ marks a subset of inactive inducible promoters in mouse embryonic stem cells. BMC Genomics 13: 424, 2012.

11. Pokholok DK, Harbison CT, Levine S, Cole M, Hannett NM, Lee TI, Bell GW, Walker K, Rolfe PA, Herbolsheimer E, et al: Genome-wide map of nucleosome acetylation and methylation in yeast. Cell 122: 517-527, 2005

12. Wang Z, Zang C, Rosenfeld JA, Schones DE, Barski A, Cuddapah S, Cui K, Roh TY, Peng W, Zhang MQ and Zhao K: Combinatorial patterns of histone acetylations and methylations in the human genome. Nat Genet 40: 897-903, 2008.

13. Mori T, Ikeda DD, Yamaguchi Y, Unoki M and Project N; NIRF Project: NIRF/UHRF2 occupies a central position in the cell cycle network and allows coupling with the epigenetic landscape. FEBS Lett 586: 1570-1583, 2012.

14. Zhang J, Gao Q, Li P, Liu X, Jia Y, Wu W, Li J, Dong S, Koseki H and Wong J: S phase-dependent interaction with DNMT1 dictates the role of UHRF1 but not UHRF2 in DNA methylation maintenance. Cell Res 21: 1723-1739, 2011.

15. Bronner C, Achour M, Arima Y, Chataigneau T, Saya H and Schini-Kerth VB: The UHRF family: Oncogenes that are drugable targets for cancer therapy in the near future? Pharmacol Ther 115: 419-434, 2007.

16. Karagianni P, Amazit L, Qin J and Wong J: ICBP90, a novel methyl $\mathrm{K} 9 \mathrm{H} 3$ binding protein linking protein ubiquitination with heterochromatin formation. Mol Cell Biol 28: 705-717, 2008.

17. Pichler G, Wolf P, Schmidt CS, Meilinger D, Schneider K, Frauer C, Fellinger K, Rottach A and Leonhardt H: Cooperative DNA and histone binding by Uhrf2 links the two major repressive epigenetic pathways. J Cell Biochem 112: 2585-2593, 2011.

18. Qian G, Jin F, Chang L, Yang Y, Peng H and Duan C: NIRF, a novel ubiquitin ligase, interacts with hepatitis $\mathrm{B}$ virus core protein and promotes its degradation. Biotechnol Lett 34: 29-36, 2012.

19. Qian G, Hu B, Zhou D, Xuan Y, Bai L and Duan C: NIRF, a novel ubiquitin ligase, inhibits hepatitis $\mathrm{B}$ virus replication through effect on HBV core protein and H3 histones. DNA Cell Biol 34 327-332, 2015

20. Lu JC, Chang YT, Wang CT, Lin YC, Lin CK and Wu ZS: Trichostatin A modulates thiazolidinedione-mediated suppression of tumor necrosis factor $\alpha$-induced lipolysis in 3T3-L1 adipocytes. PLoS One 8: e71517, 2013.
21. Sailaja BS, Cohen-Carmon D, Zimmerman G, Soreq H and Meshorer E: Stress-induced epigenetic transcriptional memory of acetylcholinesterase by HDAC4. Proc Natl Acad Sci USA 109: E3687-E3695, 2012

22. Musselman CA and Kutateladze TG: Handpicking epigenetic marks with PHD fingers. Nucleic Acids Res 39: 9061-9071, 2011.

23. He X, Duan C, Chen J, Ou-Yang X, Zhang Z, Li C and Peng H: Let-7a elevates p21(WAF1) levels by targeting of NIRF and suppresses the growth of A549 lung cancer cells. FEBS Lett 583 3501-3507, 2009.

24. Wu J, Liu S, Liu G, Dombkowski A, Abrams J, Martin-Trevino R, Wicha MS, Ethier SP and Yang ZQ: Identification and functional analysis of 9 p24 amplified genes in human breast cancer. Oncogene : 333-341, 2012.

25. Wang F, Zhang P, Ma Y, Yang J, Moyer MP, Shi C, Peng J and Qin H: NIRF is frequently upregulated in colorectal cancer and its oncogenicity can be suppressed by let-7a microRNA. Cancer Lett 314: 223-231, 2012

26. Lu S, Yan D, Wu Z, Jiang T, Chen J, Yuan L, Lin J, Peng Z and Tang H: Ubiquitin-like with PHD and ring finger domains 2 is a predictor of survival and a potential therapeutic target in colon cancer. Oncol Rep 31: 1802-1810, 2014.

27. Wu TF, Zhang W, Su ZP, Chen SS, Chen GL, Wei YX, Sun T, Xie XS, Li B, Zhou YX, et al: UHRF2 mRNA expression is low in malignant glioma but silencing inhibits the growth of U251 glioma cells in vitro. Asian Pac J Cancer Prev 13: 5137-5142, 2012.

28. Venturelli S, Armeanu S, Pathil A, Hsieh CJ, Weiss TS, Vonthein R, Wehrmann M, Gregor M, Lauer UM and Bitzer M: Epigenetic combination therapy as a tumor-selective treatment approach for hepatocellular carcinoma. Cancer 109: 2132-2141, 2007.

29. Nightingale KP, Gendreizig S, White DA, Bradbury C, Hollfelder F and Turner BM: Cross-talk between histone modifications in response to histone deacetylase inhibitors: MLL4 links histone $\mathrm{H} 3$ acetylation and histone H3K4 methylation. J Biol Chem 282: 4408-4416, 2007.

30. Luo T, Cui S, Bian C and Yu X: Uhrf2 is important for DNA damage response in vascular smooth muscle cells. Biochem Biophys Res Commun 441: 65-70, 2013.

31. Rajakumara E, Wang Z, Ma H, Hu L, Chen H, Lin Y, Guo R, Wu F, Li H, Lan F, et al: PHD finger recognition of unmodified histone H3R2 links UHRF1 to regulation of euchromatic gene expression. Mol Cell 43: 275-284, 2011.

32. Citterio E, Papait R, Nicassio F, Vecchi M, Gomiero P, Mantovani R, Di Fiore PP and Bonapace IM: Np95 is a histonebinding protein endowed with ubiquitin ligase activity. Mol Cell Biol 24: 2526-2535, 2004 